\section{DISCARDED MEDICATIONS IN VIALS ARE A SYMPTOM OF OUR HEALTH CARE SYSTEM: A CALL TO ACTION}

Recently, the National Academies of Sciences, Engineering, and Medicine (National Academies) published a congressionally mandated consensus study entitled Medications in Single Dose Vials: Implications of Discarded Drugs. ${ }^{1}$ The rationale for the study was clear: A significant volume of expensive specialty medications, such as oncology therapeutics and immunotherapies, is discarded due to variable dosing and distribution in single-use vials.

The study's overarching conclusion was that drug developers, health care providers, and payers should seek to minimize inefficiencies in drug development, delivery, and payment systems that fuel excess costs for both the health care system and for patients, rather than on trying to recoup payments for drugs that were discarded and not used in a patient's treatment.

The consensus report offered a set of interdependent recommendations to foster effective, efficient, and safe use of infused or injectable drugs. It calls for cooperation among government agencies and among nonfederal partners including manufacturers, health systems, payers, and policy-setting entities, including US Pharmacopeia (USP). The US Food and Drug Administration (FDA) and manufacturers should encourage the use of fixed dosing when it is safe to do so, rather than weight-based dosing, and discuss other ways to minimize discarded medications.

Fixed dosing is already proving valuable for certain agents (such as the tyrosine kinase and BRAF inhibitors) and monoclonal antibodies that target the immune system. For example, in 2014, FDA approved pembrolizumab, a drug used to treat several forms of advanced cancers, to be dosed based on weight. Almost 2 years later, new approvals for pembrolizumab moved from weight-based to fixed dosing. Currently, pembrolizumab is given with fixed dosing for all indications. ${ }^{2}$ The Secretary of the Department of Health \& Human Services (HHS) should direct FDA, Centers for Medicare \& Medicaid Services, and Centers for Disease and Prevention to work with USP, joined by pharmacists and infectious disease experts, to harmonize guidelines on vial optimization. Pharmacist experts will be called on to provide guidance on feasible, evidence-based approaches regarding dosing, compounding, stability, medication administration, and use protocols to safely use single-dose vials for more than 1 patient. Lessons can be learned from other advanced countries that have identified clinical strategies and technological approaches for safely and effectively sharing vials through bolstered industrial design and systems engineering. ${ }^{3}$ HHS should also prioritize efforts to require manufacturers to produce medications in multidose vials when it is safe to do so.

With the current rollout of the COVID-19 vaccines, this consensus report's focus on effectively, efficiently, and safely using infused or injectable drugs is timely. The societal benefit of fixed dosing per patient in a multidose vial has been demonstrated. Accordingly, calls for increasing the number of medications in multidose vials have increased. The Hematology/ Oncology Pharmacy Association, in a position statement, called on manufacturers to increase medications available in multidose formulations so as to reduce discarded medications. ${ }^{4}$

We must reconsider how we produce, dose, and administer these infused or injectable medications. As the availability and prices of specialty medications continue to increase, it is imperative that we also adjust policies aimed at producing and administering these drugs, seeking to ensure access, reduce waste, and maximize supply for patients.

\author{
Jonathan $\mathrm{H}$ Watanabe, PharmD, PhD \\ Associate Dean of Assessment and \\ Quality \\ Professor of Clinical Pharmacy \\ Department of Clinical Pharmacy \\ Practice \\ University of California Irvine School \\ of Pharmacy \& Pharmaceutical \\ Sciences \\ Irvine, CA \\ jonathan.watanabe@uci.edu \\ Tracy Lieu, MD, MPH \\ Director, Division of Research \\ Kaiser Permanente \\ Northern California \\ Oakland, CA \\ Edward H Shortliffe, MD, PhD \\ Chair Emeritus and Adjunct Professor, \\ Department of Biomedical Informatics \\ Vagelos College of Physicians \\ and Surgeons, Columbia University \\ New York, NY
}

\section{DISCLOSURES}

No funding was received for the writing of this letter. All authors were members of the National Academies study committee to which this letter refers, with Shortliffe as the chair. All committee members who contributed to the report were themselves vetted for potential conflicts of interest, as is described in the recently published version of the report. Lieu is an employee of Kaiser Permanente and the content does not represent the official views of Kaiser Permanente. The other authors have nothing to disclose. 


\section{REFERENCES}

1. National Academies of Sciences, Engineering, and Medicine. Medications in Single-Dose Vials: Implications of Discarded Drugs. The National Academies Press; 2021. https://doi. org/10.17226/25911.

2. US Food and Drug Administration. FDA approves new dosing regimen for pembrolizumab. April 29, 2020. Accessed March 11, 2021. https://www.fda.gov/ drugs/drug-approvals-and-databases/ fda-approves-new-dosing-regimen-pembrolizumab
3. Gilbar PJ, Chambers CR,

Vandenbrouche J, Sessink PJ, Tyler TG.

How can the use of closed system transfer devices to facilitate sharing of drug vials be optimised to achieve maximum cost savings? J Oncol Pharm Pract. 2019;25(1):205-09. doi:10.1177/1078155217753890
4. Hemotology/Oncology Pharmacy Association. Intravenous Cancer Drug Waste Issue Brief. HOPA Position Statement. 2019. Accessed March 11, 2021. https://www.hoparx.org/images/ hopa/advocacy/Issue-Briefs/Drug Waste_2019.pdf 